\title{
Transitive Inference after Minimal Training in Rhesus Macaques (Macaca mulatta)
}

\author{
Greg Jensen ${ }^{1,4}$, Fabian Munoz ${ }^{1,4}$, Anna Meaney ${ }^{1,4}$, Herbert S. Terrace ${ }^{2,3}$, and Vincent $P$. \\ Ferrera $^{1,3,4}$
}

\author{
${ }^{1}$ Dept. of Neuroscience, Columbia University Medical Center \\ ${ }^{2}$ Dept. of Psychology, Columbia University \\ ${ }^{3}$ Dept. of Psychiatry, Columbia University Medical Center \\ ${ }^{4}$ Zuckerman Mind Brain Behavior Institute, Columbia University
}

\section{ABSTRACT}

Rhesus macaques, trained for several hundred trials on adjacent items in an ordered list (e.g. $A>B$, $B>C, C>D$, etc.), are able to make accurate transitive inferences ( $\mathrm{TI}$ ) about previously untrained pairs (e.g. $A>C, B>D$, etc.). How that learning unfolds during training, however, is not well understood. We sought to measure the relationship between the amount of training and the resulting response accuracy in four rhesus macaques, including the absolute minimal case of seeing each of the six adjacent pairs only once prior to testing. We also ran conditions with 24 and 114 trials. In general, learning effects were small, but they varied in proportion to the square root of the amount of training. These results suggest that subjects learned serial order in an incremental fashion. Thus, rather than performing transitive inference by a logical process, serial learning in rhesus macaques proceeds in a manner more akin to a statistical inference, with an initial uncertainty about list position that becomes gradually more accurate as evidence accumulates.

Keywords: transitive inference, serial learning

Transitive inference (TI), the ability to infer $\mathrm{A}>\mathrm{C}$, given the premises $\mathrm{A}>\mathrm{B}$ and $\mathrm{B}>\mathrm{C}$, has been observed in many species. The empirical evidence that rhesus monkeys can succeed at TI tasks is unambiguous (reviewed by Jensen, 2017), and multiple lines of evidence point to this inference being accomplished using a representational, model-based framework (Jensen et al., 2013, 2019). The natural consequence of this line of theorizing is the suggestion that the process that monkeys are engaged in can be characterized as an informal kind of statistical inference, refining their model of an ordered list as additional evidence presents itself.

Most studies of learning consist of extended periods of training, followed by a rigorous analysis of the resulting asymptotic behavior. For example, Wynne (1997) collected between 11,000 and 17,000 responses per subject, a relatively common approach in studies using pigeons. Training that extends over tens of thousands of trials is also common studies of rhesus macaques, a problem that complicates comparison of non-human performance with that of human participants (Stoet and Snyder, 2007). Extended training of this kind often arises when experimental designs require subjects to cross some performance threshold (e.g. at least $70 \%$ accuracy) before they can advance to the next phase. A consequence of this is that such studies reveal less about the process by which learning unfolds than they do about the end results of those processes.

Other studies, however, demonstrate that learning can be quite rapid, with behavioral signatures emerging after merely tens or hundreds of responses. Rhesus macaques (Jensen et al., 2015) and two species of lemur (Tromp et al., 2015) performing TI tasks display robust learning after fewer than 250 trials. 
In the related Simultaneous Chain serial learning paradigm, rhesus macaques displayed clear learning effects in under 40 trials (Jensen et al., 2013).

To this end, we sought to measure the effect of training on the acquisition of representations of ordered lists, with an emphasis on very brief training intervals. Rather than studying how well subjects could learn any given ordered list of stimuli given exhaustive training, we reduced training to the theoretical minimum of one exposure per adjacent pair (a total of six training trials). Because exposure to each stimulus set was brief, we were able to test each NHP with hundreds of novel lists, providing sufficient statistical power to characterize the early learning displayed by each subject and to detect even small behavioral differences.

\section{METHODS}

\section{Subjects}

Subjects were four adult male rhesus macaques (Macaca mulatta), N, O, R, and S. All four subjects had extensive prior experience with transitive inference procedures. However, the stimuli used in this experiment were novel to these subjects, having not been used in any previous experiments.

Subjects performed the experimental task in their home cage while housed individually in a colony room containing approximately a dozen macaques. Training took place five days a week, one session each day. In order to maintain motivation, subjects were fluid-restricted (300mL of water a day) two days prior to the first day of testing. Depending on task performance, subjects could earn up to 500mL a day depending on performance, with $250 \mathrm{~mL}$ to $350 \mathrm{~mL}$ being typical. Most days, subjects earned their entire fluid ration performing the task. This was supplemented as needed after the session to meet the minimum requirement. Each monkey received biscuits prior to testing, and fresh fruit following testing. These protocols were approved by the Institutional Animal Care and Use Committee (IACUC) at Columbia University, in accordance with the guidelines provided by the Guide for the Care and Use of Laboratory Animals of the National Institute of Health (NIH).

\section{Apparatus}

The task was performed using a cage-mounted touchscreen tablet that could be installed and removed in the subject's home cage each day. Stimuli were presented on a 10.1" HD capacitive touch screen (1366 x 768 resolution), upon which subjects made manual responses. Each correct response yielded $0.75 \mathrm{~mL}$ water rewards, delivered to a spigot through a solenoid valve by a gravity-fed 1L bottle attached to the exterior of the cage. The task was programmed using JavaScript and run in a Google Chrome browser (set to kiosk mode) under a Windows 8.1 operating system with NodeJS acting as a local fileserver. Details of the apparatus are described by Tanner et al. (2015).

\section{Procedure}

Subjects performed a transitive inference task using ordered 7-item lists, with their order denoted by the letters ABCDEFG. Figure 1A provides an example of one such ordered list. After touching a start stimulus to begin the trial, subjects were shown pairs of images at a time (e.g. the pair BC, consisting of the second and third images in the list), and were rewarded for selecting the item with a lower rank. For example, selecting stimulus B was rewarded when presented with the pair BC, but was not rewarded when presented with the pair AB. The trial structure and on-screen stimulus arrangement is depicted in Figure $1 \mathrm{~B}$.

Each session consisted of a total of 540 trials, arranged into alternating training and testing phases. During each training phase, a new set of 7 stimuli was introduced that the subject had never been exposed to previously, and they were presented with only the adjacent pairs from that list $(\mathrm{AB}, \mathrm{BC}, \mathrm{CD}, \mathrm{DE}, \mathrm{EF}$, 

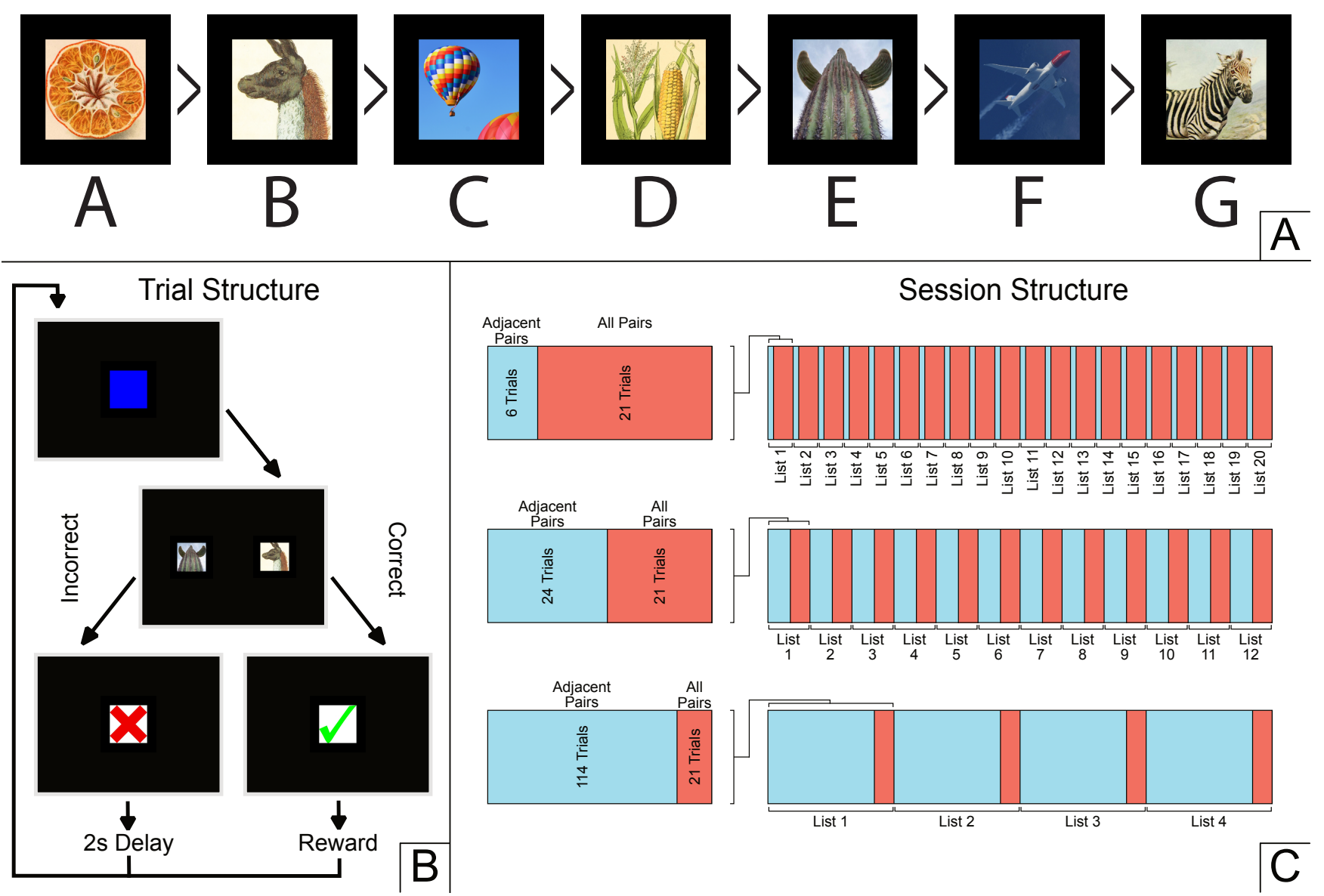

Figure 1. Experimental procedure. A. Example of a seven-item list, with stimuli labeled A through G. Note that although all seven stimuli are used throughout the experiment, only two are visible during any given trial. B. Structure of an experimental trial. Subject touched the blue start stimulus, which was then replaced by a pair of images from the current list. A correct response (to the item with an earlier list position) yielded a green check mark and a reward. An incorrect response yielded a red $\mathrm{X}$ and a short timeout before the start of the next trial. C. Overall session structure, in which each session was divided into a separate section for each list learned that day, and each section was further divided into a training phase (in light blue) and a testing phase (in red). Training phases varied in their duration, but testing phases always consisted of 21 trials.

and FG). The training phase came in one of three durations: it either lasted 6 trials (showing each adjacent pair once), 24 trials (showing each pair four times), or 114 trials (showing each pair 19 times). Then, during the following testing phase, subjects were presented with each of the 21 possible pairs exactly once each. Once the 21-trial testing phase was completed, subjects never saw that set of stimuli again. Sessions always used identical training durations, so a 540-trial session consisted of either 20 cycles of training \& testing (for 6-trial training phases), or 12 cycles (for 24-trial training), or 4 cycles (for 114-trial training). A novel set of 7 images was used for each training/testing cycle. On-screen presentation of stimuli was randomized, with rewarded stimuli appearing on the left and right sides of the screen with equal frequency; however, because of the small number of trials in each phase, stimulus positions were not systematically counterbalanced. This overall session structure is depicted in Figure 1C.

Because subjects did not always finish all phases in a particular session, the total number of lists to which they were exposed varied. Subject $\mathrm{N}$ was exposed to 800 different lists during 6-trial training, 240 lists during 24-trial training, and 108 lists during 114-trial training. Subject O was exposed to 787 lists 
during 6-trial training, 228 lists during 24-trial training, and 71 lists during 114-trial training. Subject $\mathrm{R}$ was exposed to 805 lists during 6-trial training, 237 lists during 24-trial training, and 62 lists during 114-trial training. Subject $\mathrm{S}$ was exposed to 803 lists during 6-trial training, 226 lists during 24-trial training, and 58 lists during 114-trial training.

\section{Analysis}

Previous studies of transitive inference have often relied on logistic regression as a way of estimating the probability of a correct response directly (refs). Consider, for example, the following generalized linear model:

$$
p(\text { Correct })=\left(1+\exp \left(-\left(\beta_{\emptyset}+\beta_{t} \cdot t\right)\right)\right)^{-1}
$$

Here, the parameter $\beta_{\emptyset}$ governs response accuracy at the start of the experiment ("trial zero") by acting as an intercept term, and the parameter $\beta_{t}$ governs how accuracy grows as a function of the trial number $t$, acting as a slope term. In conventional TI tasks, subjects are exposed to the same set of stimuli for hundreds or thousands of trials, ensuring that they are able to achieve at least moderate levels of response accuracy for any given list. Furthermore, most experimental designs counterbalance stimulus positions in order to ensure that subjects have an incentive to minimize spatial response bias. Since such designs try to maximize the estimated effect size of learning, Equation 1 generally provides a reliable description of performance. Since learning generally has a much larger effect on performance than response bias, and since a counterbalanced design tends to reduce such bias, we have generally found that controlling for response bias reduces statistical power without much benefit in capturing the effect of learning.

Although this study follows the basic design of other TI studies, the small number of training trials virtually guarantees that any observed effect sizes due to learning will also be small. This makes controlling for response bias much more important, as the presence of bias could much more easily swamp any learning effects. The lack of counterbalancing of stimulus position, a concession made to make each learning phase as brief and unpredictable as possible, potentially exacerbated this bias problem. To this end, we sought to use an interaction model to estimate response accuracy by factoring it out of a model designed to predict the probability of making a response to the stimulus on the left (McElreath, 2020). To capture the effects of learning and response bias during training, such a model takes the following form:

$$
p(\text { Left })=\left(1+\exp \left(-\left(\beta_{L}+\beta_{L t} \cdot t+L\left(\beta_{\emptyset}+\beta_{t} \cdot t\right)\right)\right)\right)^{-1}
$$

Here, $\beta_{L}$ corresponds to a subject's initial bias to choose the stimulus on the left side of the display at trial zero (the intercept), whereas $\beta_{L t}$ shows how the bias changes over time (the slope). The variable $L$ is a centered dummy-code that has a value of 0.5 when the stimulus on the left is a correct response, and a value of -0.5 when the left stimulus is incorrect. As a result, $\beta_{\emptyset}$ and $\beta_{t}$ retain their original interpretation: They provide intercept and slope estimates of response accuracy having accounted for the response bias estimated by $\beta_{L}$ and $\beta_{L t}$. Given the large sample size of different training lists, this model has the potential to detect very small learning effects by factoring out the most important source of bias that might obscure those effects.

A similar model was used to for the all-pairs testing phase. Here, trials were removed as a predictor, with symbolic distance $D$ instead used as a predictor of response accuracy:

$$
p(\mathrm{Left})=\left(1+\exp \left(-\left(\beta_{L}+L\left(\beta_{\emptyset}+\beta_{D} \cdot D\right)\right)\right)\right)^{-1}
$$



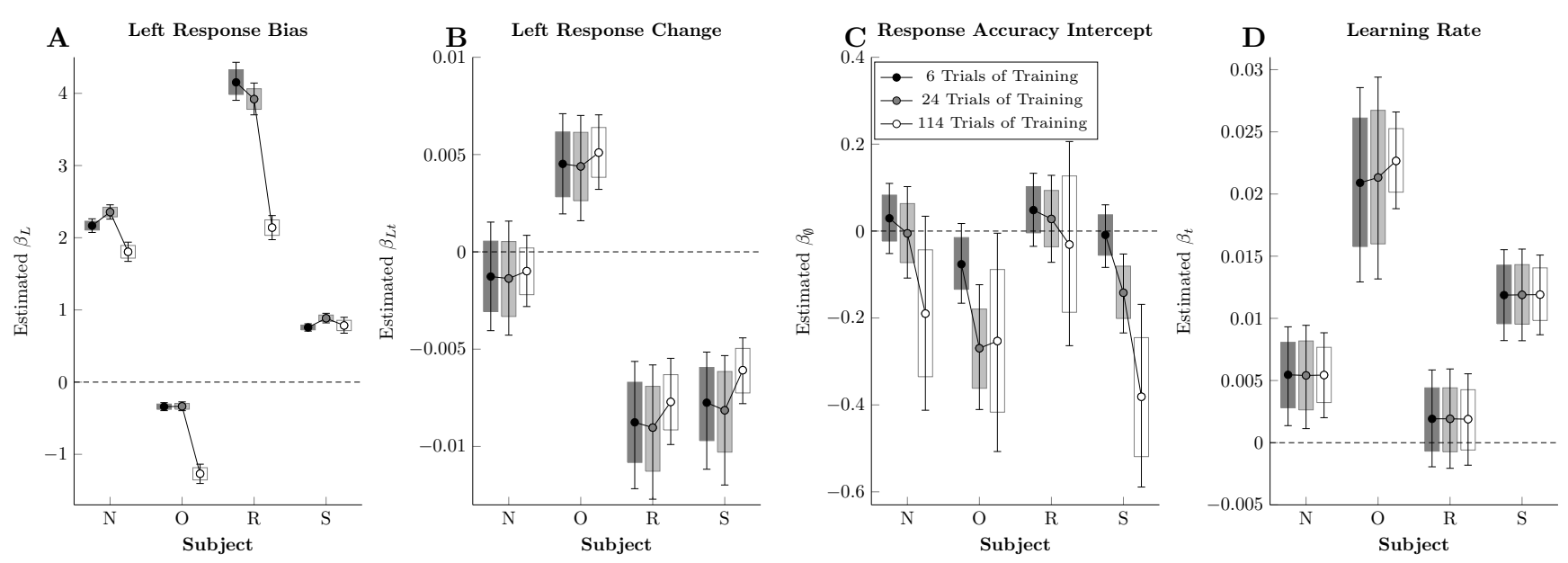

Figure 2. Model parameter estimates for each subject's performance during training, using Equation 2. All parameters are estimated in log-odds units. Boxes correspond to the $80 \%$ credible interval for each estimate, while whiskers correspond to the 95\% credible interval. A. Estimate of left response bias at the start of the training phase. B. Estimate of the rate of change in left response bias per trial. C. Estimate of the interaction parameter governing response accuracy at the start of the training phase. D. Estimate of the interaction parameter governing the change in response accuracy per trial during training.

In order for $\beta_{\emptyset}$ to retain its interpretation as an indicator of "overall response accuracy," $D$ is centered by giving each case a value of its symbolic distance minus a value of $\frac{8}{3}$, the mean distance across the 21 pairs. The parameter $\beta_{D}$ therefore most properly corresponds to a subject's deviation from baseline accuracy as a function of that pair's deviation in its symbolic distance.

Parameters in Equations 2 and 3 were fit using the Stan programming language (Carpenter et al., 2017) as multi-level models. This hierarchy represented subjects as belonging to a population, but also modeled individual lists as a population of parameters for each subject, effectively making the model a three-tiered hierarchy. This allowed for the possibility that a subject's parameters could vary from one list of items to the next, rather than presuming that behavior generated by a particular subject across all lists were governed by the same parameter distribution. Overall means and uncertainties for each subject were then marginalized out of the resulting posterior distribution. Additionally, Equation 3 was evaluated for each condition separately.

\section{RESULTS}

Figure 2 plots the mean subject-level parameter estimates for the regression parameters in Equation 2. The most noteworthy consequence of this analysis is that subjects displayed a much stronger spatial bias than we had anticipated when designing the experiment (as measured by values of $\beta_{L}$ far from zero), particularly subjects $\mathrm{N}$ and $\mathrm{R}$. That was likely due in part to the lack of counterbalancing, but may also have been a result of overall task difficulty. In the 6-trial and 24-trial training sessions, most responses were made to stimuli to which subjects had very little exposure before getting switched to a new list. This may have resulted in low motivation to attend to the task. However, bias tended to go down over successive trials (as revealed by values of $\beta_{L t}$ of an opposite sign to the initial bias), and three of the four subjects displayed a consistently positive mean learning rate (as measured by $\beta_{t}$ ) across all levels of training.

The implications of these results are shown in Figure 3, which plots the probability of a left choice over consecutive blocks of trials. The effect of serial learning is not the increase in this probability (which is a form of bias), but rather the separation of the "left is correct" cases (blue filled points) and the "left is incorrect" cases (red open points). By the end of the 114-trial training phases, a clear separation between 
Subject N

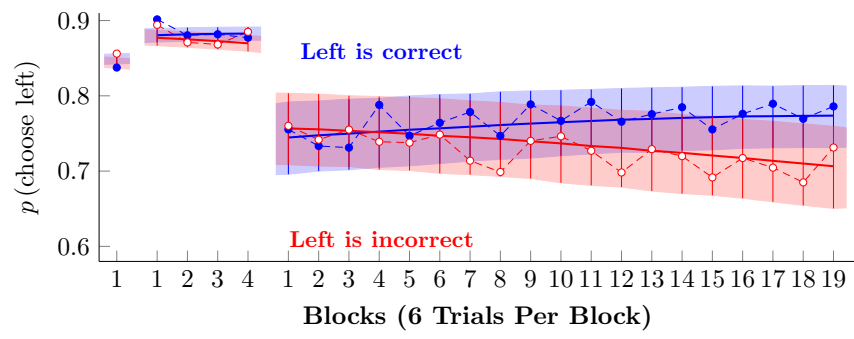

Subject $\mathbf{R}$

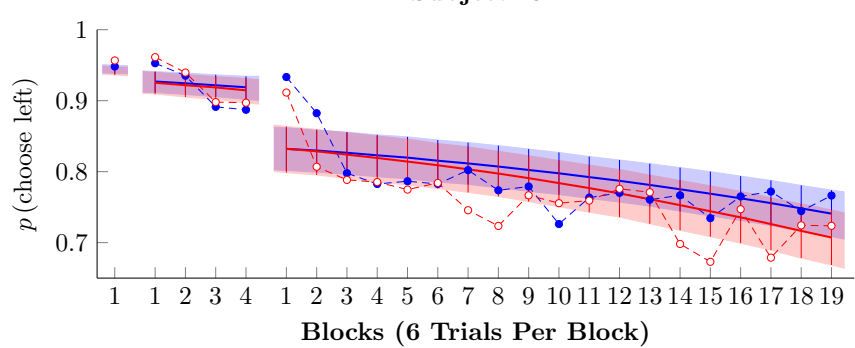

Subject O

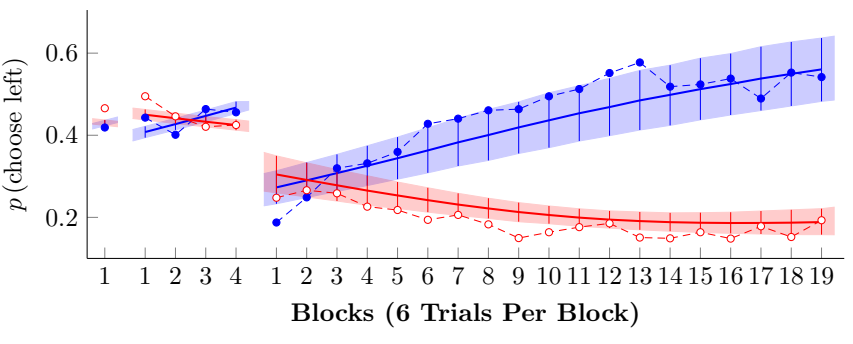

Subject S

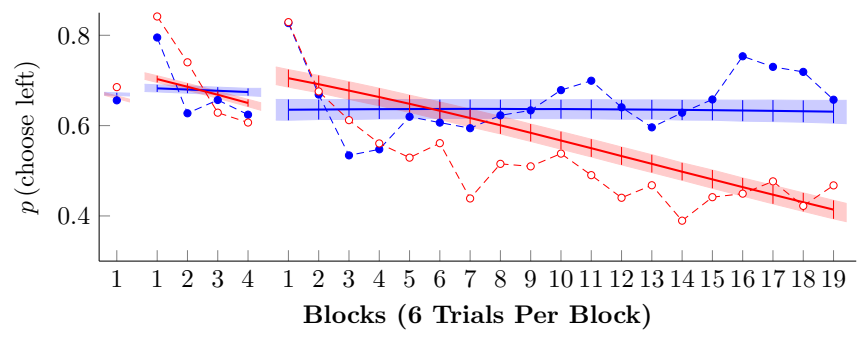

Figure 3. Estimated probability of a left response over consecutive blocks of training ( 6 trials per block), divided by whether a left response was correct (in blue, filled points) or incorrect (in red, white points). The shaded region for each estimate corresponds to the $95 \%$ credible interval of the estimate.

correct and incorrect left responses has emerged in subjects $\mathrm{O}$ and $\mathrm{S}$, while the initial hints of a separation are beginning to manifest (but are not yet robust) in subjects $\mathrm{N}$ and $\mathrm{R}$. However, low accuracy during training phases in TI tasks is not necessarily reflective of a lack of learning in monkeys, who frequently do poorly on adjacent pairs even after thousands of training trials, only to show higher accuracy and clear symbolic distance effects as soon as the task switches to an all-pairs testing phase.

Subjects' ability to transfer their training experience into test-phase accuracy is conveyed by the parameter estimates in Figure 4. As in Figure 2, subjects displayed clear left-stimulus biases during the testing phase. However, having controlled for that bias, the overall response accuracy during testing was above chance for all subjects in all conditions (as measured by $\beta_{\emptyset}$ ). Furthermore, the stronger the measured learning effect at the end of training, the larger the benefit of that training for response accuracy during testing. Symbolic distance effects were reliable observed in two subjects (as measured by $\beta_{D}$ ), and the parameter estimates for the other two subjects were generally positive.

The implications of this analysis are plotted in Figure 5, which like Figure 3 plots a subject's probability of choosing the left stimulus, then splits performance into the cases where the left response is either correct (blue filled points) or incorrect (red open points). These effects are often quite small (on the order of around a 4\% difference in subjects $\mathrm{N}$ and $\mathrm{R}$ ), but the separations are nevertheless clear in the 6-trial case The small effect sizes could be reliably estimated because each estimate is the result of hundreds of lists of evidence. The cases with longer training are sometimes more uncertain because they are aggregated across fewer lists, despite having larger effect sizes.

Describing behavior only in terms of an intercept and a distance effect does not shed much light on the specific inferences being made. To address this, another analysis was performed in which each of the 21 possible test pairs was given its own parameter, without explicitly encoding symbolic distance as a predictor. As such, each pair during testing was modeled using the follow equation:

$$
p(\operatorname{Left})=\left(1+\exp \left(-\left(\beta_{L}+L\left(\beta_{\emptyset}\right)\right)\right)\right)^{-1}
$$



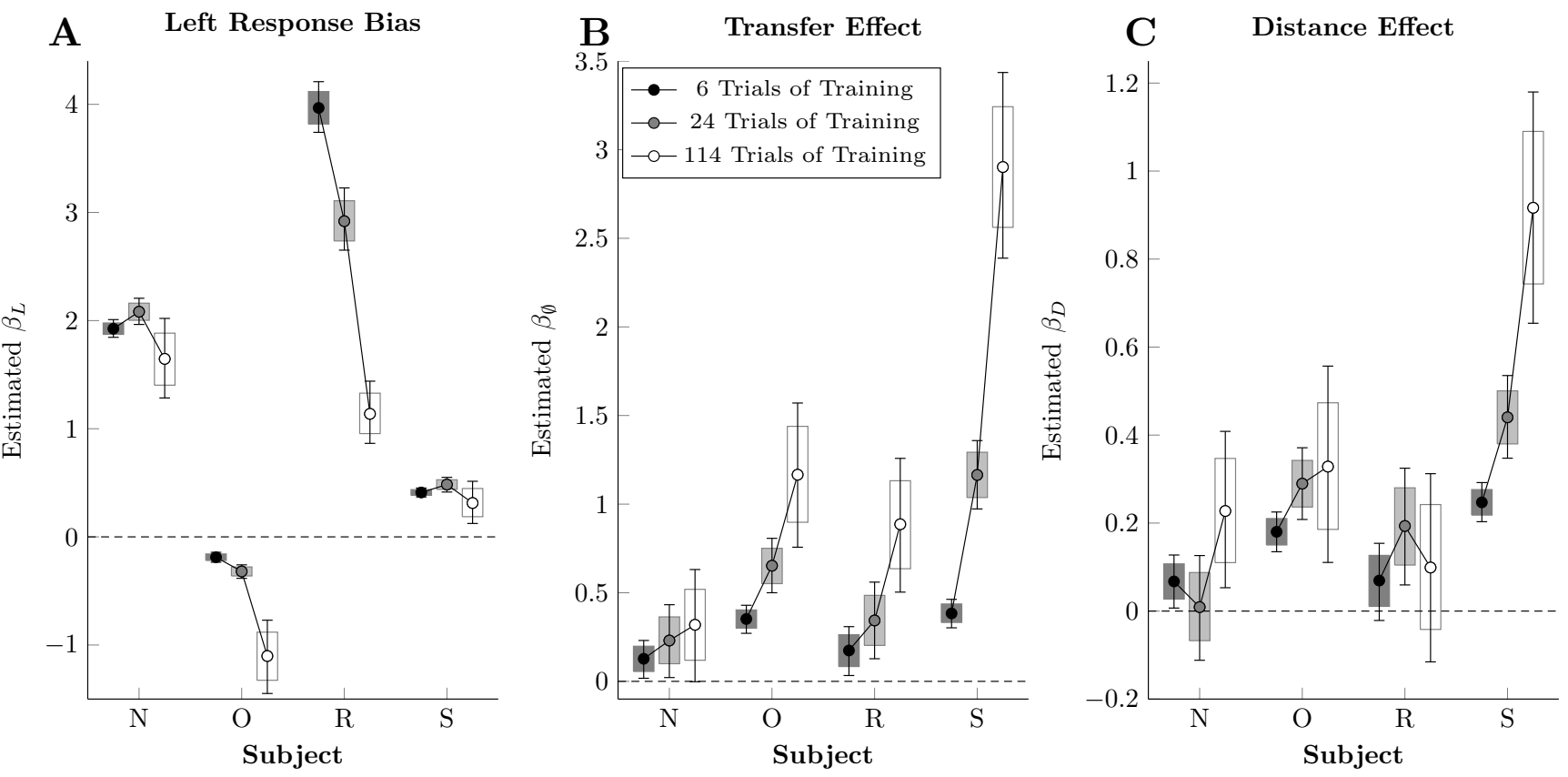

Figure 4. Model parameter estimates for each subject's performance during testing, using Equation 3. All parameters are estimated in log-odds units. Boxes correspond to the $80 \%$ credible interval for each estimate, while whiskers correspond to the 95\% credible interval. A. Estimate of left response bias during testing. B. Estimate of the interaction parameter governing response accuracy during testing. C. Estimate of the interaction parameter governing the symbolic distance effect during testing.

Subject N
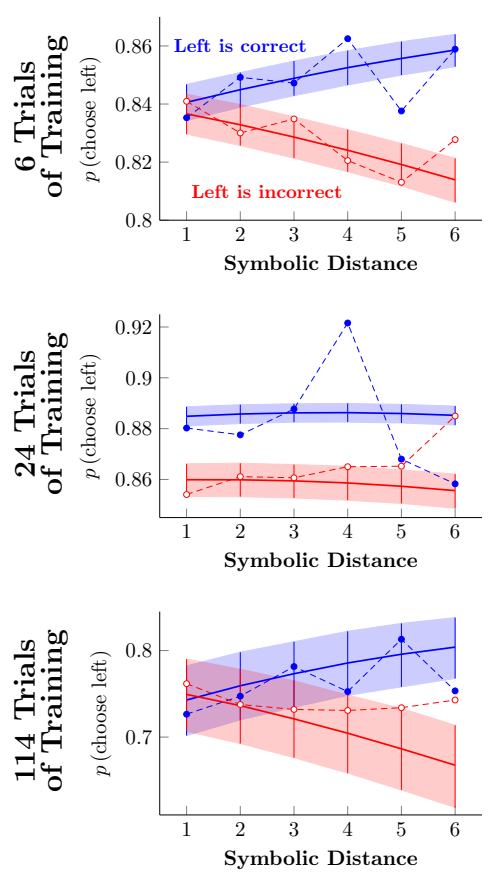

Subject O
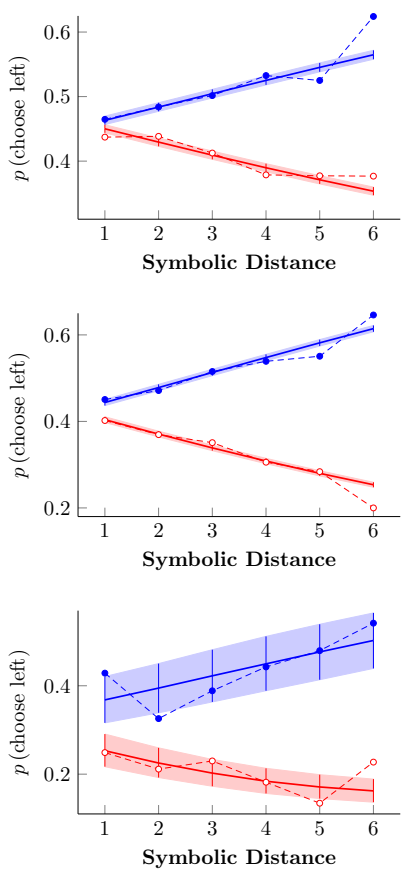

Subject R
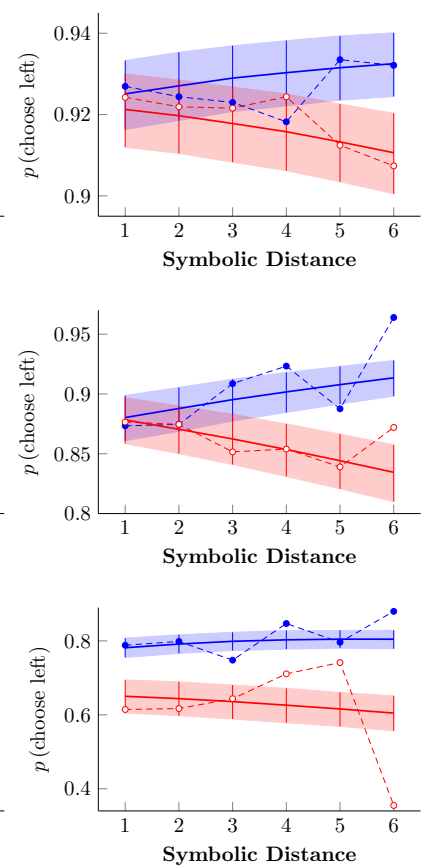

Subject $\mathbf{S}$
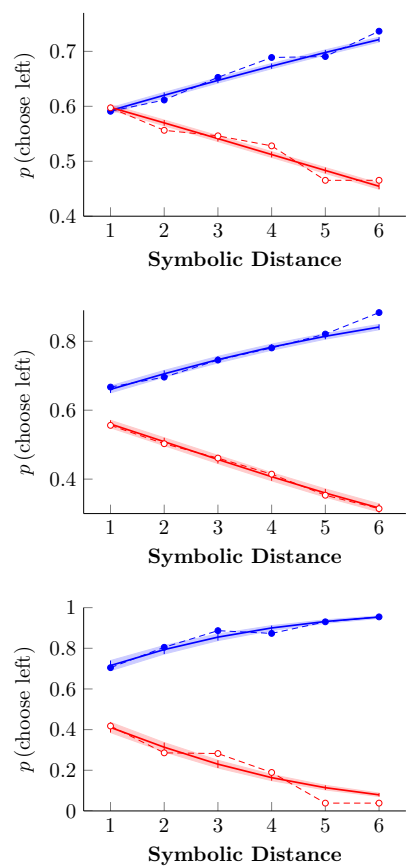

Figure 5. Estimated probability of a left response across the six different symbolic distances, divided by whether a left response was correct (in blue, filled points) or incorrect (in red, white points). The shaded region for each estimate corresponds to the $95 \%$ credible interval of the estimate. 

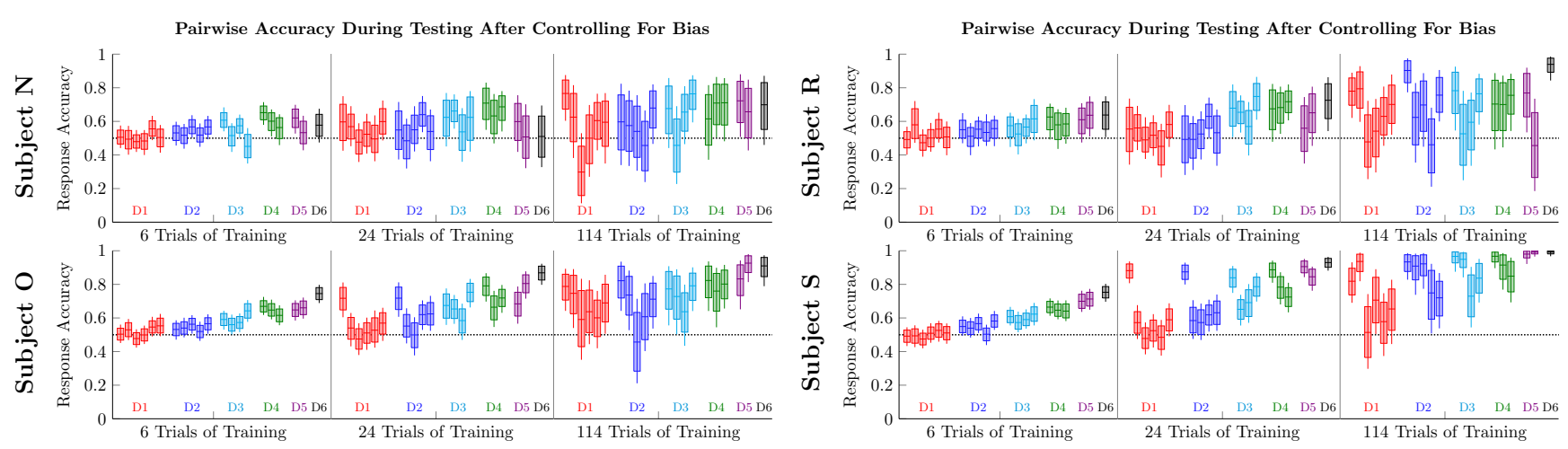

Figure 6. Counterfactual estimates of what response accuracies would have been during testing has subjects displayed no left response bias, evaluated independently for each pair and for each condition using Equation 4. Boxes correspond to the $80 \%$ credible interval for each estimate, while whiskers correspond to the $95 \%$ credible interval.

As in Equations 2 and 3, the left-response bias was measured by $\beta_{L}$, and the response accuracy was measured by an interaction term $\beta_{\emptyset}$. The testing phases for each of the three training conditions $(6,24$, or 114 trials) and for each of the 21 pairs were analyzed independently.

Response accuracy for each of the 21 pairs is shown in Figure 6. Left-response bias is factored out of these estimates. That is, the response accuracies in Figure 6 presume that $\beta_{L}$ was set to a value of 0.0 , in order to help make the differences in behavior clearer. Even though symbolic distance was not encoded in this analysis, the pairwise accuracy in all subjects nevertheless appears to depend on symbolic distance in a roughly linear fashion, even when there were only 6 trials of training (Figure 6, left column). The main qualitative difference between the minimal 6-trial training case and longer periods of training is that subjects in the minimal case do not show signs of a terminal item effect, and such effects only begin to emerge after longer periods of training. Additionally, although 114 trials of training were sufficient for most subjects to consistently exceed chance for at least some pairs, all subjects had at least a few pairs whose accuracy overlapped with chance (and subjects $\mathrm{N}$ and $\mathrm{R}$ overlapped with chance for more than half of the test pairs).

By estimating each of the 21 possible pairs separately, we were able to determine whether the "assumption of scalar distance" made by Equation 3 was valid. In other words, we can test whether the symbolic distance effect, or its internal representation, is linear. Using the approach described by Jensen et al. (2013), we converted the probabilities in Figure 6 to scalar values using a logit transform, and then estimated the mean distance of each stimulus to every other stimulus in terms of log-odds units. As such, the response accuracy for any pair of stimuli could be obtained by subtracting one "position estimate" from the other and subjecting that difference to a logistic transformation.

Figure 7 plots these estimates of stimulus position. These results confirm that subjects, on average, reliably behaved in a manner consistent with stimuli being approximately evenly spaced along a continuum after 6 trials of training. One effect of longer training was to increase the steepness of the item position $\mathrm{v}$. stimulus function, as if the internal spacing of the items tended to increase with training. Wider spacing in log-odds units decreases the confusability of the stimuli, thereby increasing overall accuracy. Interestingly, however, although the spacing of non-terminal items B through F remained roughly linear over longer training periods, the gains in performance accrued between 24 and 114 trials seemed mainly driven by the terminal items A and G. Thus, although subjects displayed a pattern of responding consistent with learning the overall ordering of the stimuli, what emerged over the course of training was a differential learning rate for terminal items, which manifest as a substantial terminal item effect on top of a slower background rate of serial learning. 

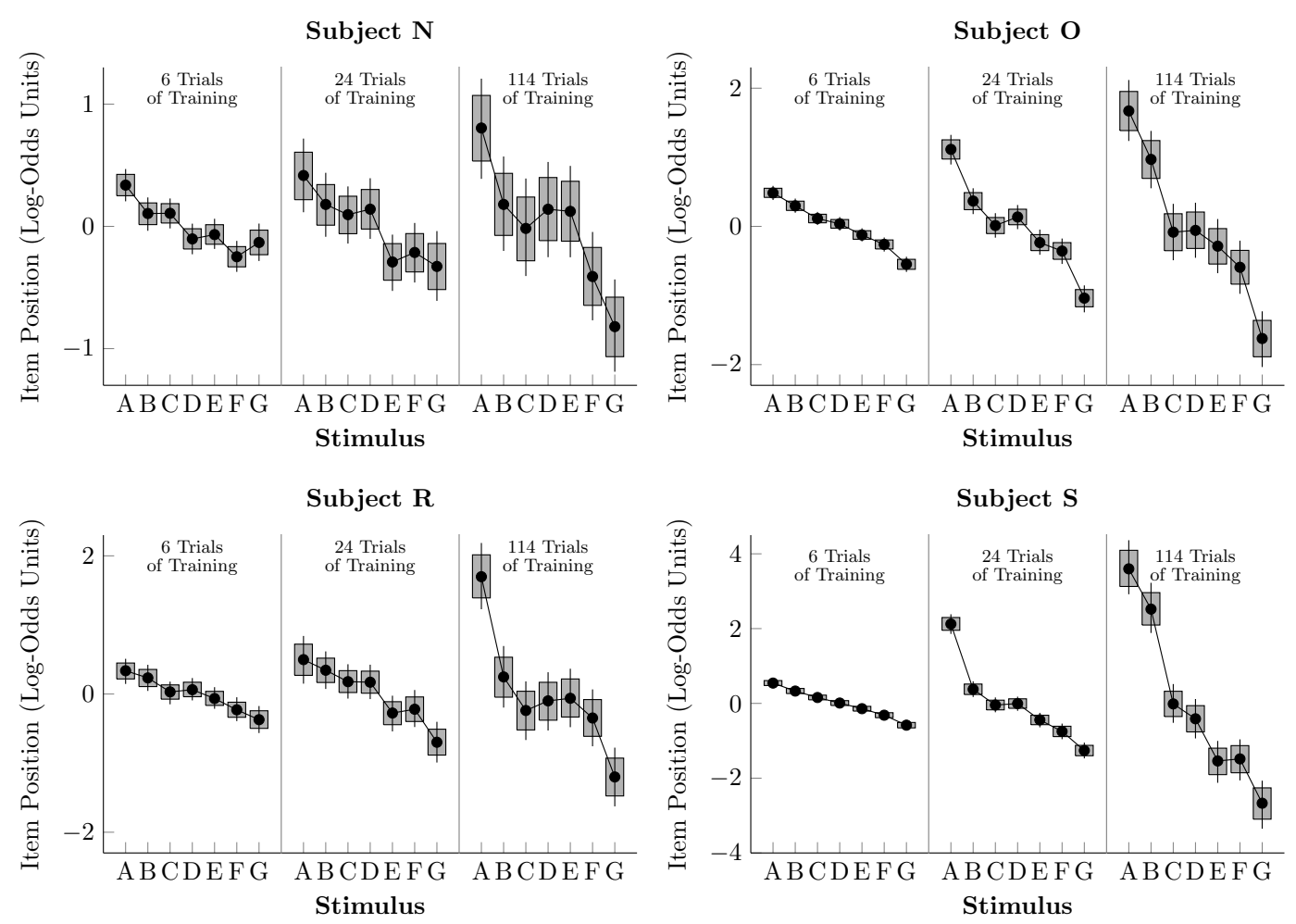

Figure 7. Estimates of "item position" derived from response accuracies in Figure 6 for each condition. Positions are measured in log-odds units, so the accuracy of any given pair is given by the logistic transform of the difference between any two items. Boxes correspond to the $80 \%$ credible interval for each estimate, while whiskers correspond to the $95 \%$ credible interval.

\section{DISCUSSION}

Subjects performed a TI task in which they received varying amounts of training on the adjacent pairs before being exposed to the full testing set of 21 possible pairs. The experiment included the theoretically minimal case of one exposure to each adjacent pair during training (6 trials), and one exposure to each pair during testing (21 trials). The short training and testing cycle allowed subjects to be exposed to many lists per session. Any learning effects are expected to be small, but were detectable due to the massive size of the dataset. Despite the brevity of this training paradigm, subjects reliably showed evidence of learning (Figs. $2 \& 3$ ) and successfully transferred their knowledge of the ordering from the training pairs to the testing pairs, displaying transitive inferences even after only 6 trials of training (Figs. $4 \& 5$ ). The results demonstrate that a symbolic distance effect (SDE), one of the hallmarks of serial learning, was evident after minimal training. Another oft-reported feature of serial learning, the terminal item effect (TIE), emerged only after more extended training.

In order to better understand what such rapid inferences look like in practice, performance for each of the 21 testing pairs was fit separately for each training condition (Figure 6), and these estimated response accuracies had a structure consistent with an initially linear orderings of stimuli in all conditions whose terminal items became more clearly dissociated from the non-terminal items over the subsequent trials of training (Figure 7). This pattern of errors, including the resulting terminal item effect, was more consistent with a model-based learning process than it was with a model-free process based on prediction error (Figure 8). 

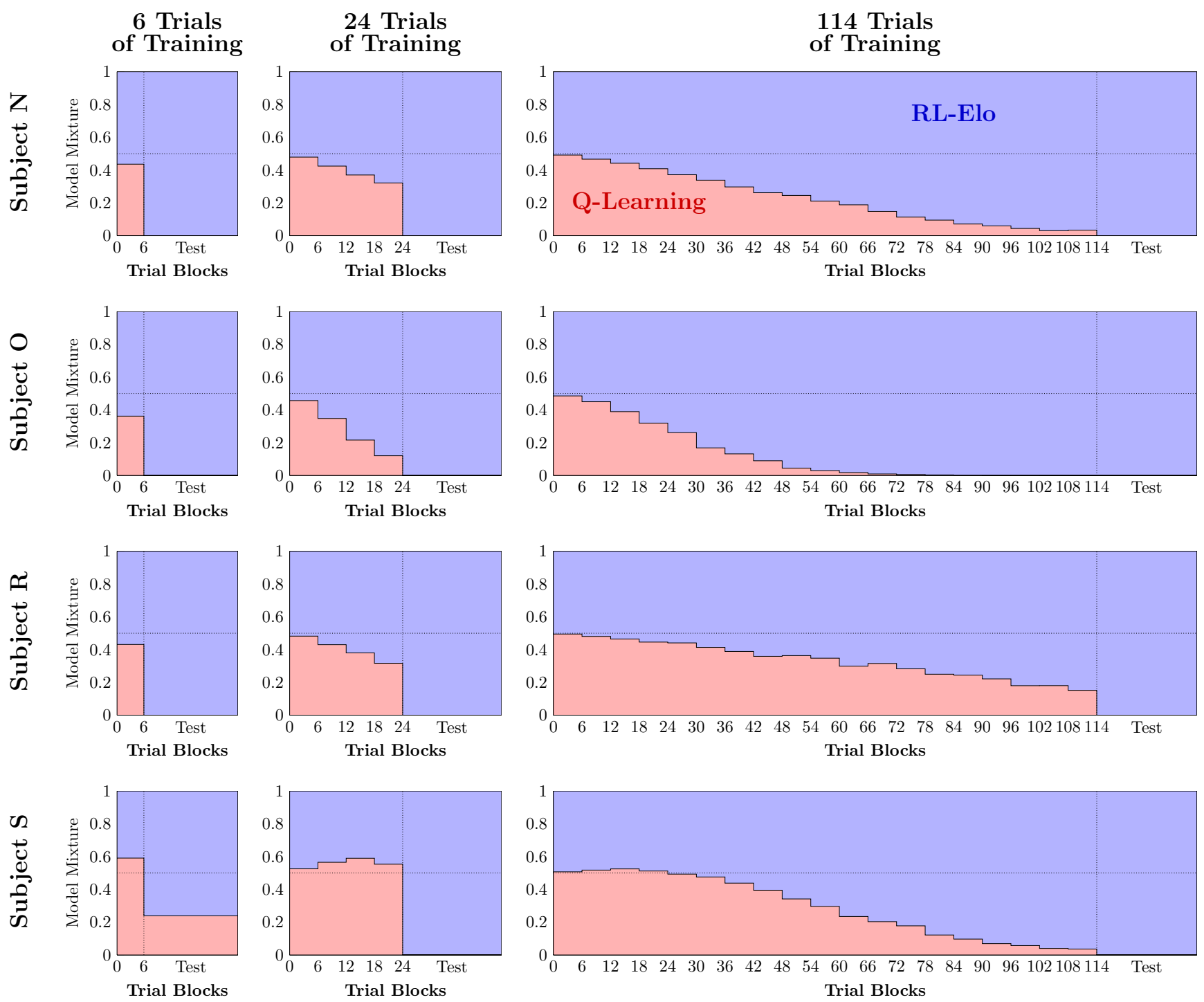

Figure 8. Ensemble model comparing the efficacy of the RL-Elo algorithm (in blue, above) to the $Q$-learning algorithm (in red, below) over consecutive trial blocks in each condition. The proportion of the model mixture was obtained by fitting each model using maximum likelihood parameters, and using the resulting BIC scores for each model as a proxy for the log-likelihood.

\section{Theoretical Implications for Serial Learning}

What sort of computational process might underlie such learning? Broadly, most accounts of TI fall into one of two camps: Either model-free learning processes that rely on reward prediction error, or model-based learning processes that use task feedback to update an internal representation of the stimulus ordering. Model-free algorithms are less effective than model-based approaches at explaining behavioral correlates of TI across a wide range of experimental designs, but no single approach captures the full range of phenomena that have been observed empirically. Furthermore, both approaches often make similar predictions. For example, the terminal item effect is expected from most model-free processes, because the terminal items have differential reward rates when compared to other stimuli. However, some model-based processes also predict terminal item effects, because there is less ambiguity about the information provided by those stimuli. To evaluate whether the error patterns displayed (Figure 6) and the ordering of stimuli that they imply (Figure 7), we modeled subjects' behavior using two different approaches that both predict 
terminal item effects. Our model-free algorithm was $Q$-learning (Watkins and Dayan, 1989), which is analogous to a Rescorla-Wagner associative learning model. Our model-based approach was RL-Elo (Kumaran et al., 2016), which models stimuli positions using difference in log-odds position values of the same sort as depicted in Figure 7.

Following the quadratic approximation approach described by Jensen et al. (2019), we fit subjects' response data using maximum likelihood model parameters and generated an ensemble prediction that consisted of a weighted combination of the two models. Model weights were determined by an inverse logistic transformation of the difference between their respective BIC scores. Figure 8 shows the resulting mixture of the two models over the course of training. In all subjects, $Q$-learning and RL-Elo yielded comparable performance during the first few training blocks, but RL-Elo's ability to predict subject performance became progressively more dominant as training progressed, until subject performance at trial 114 was overwhelmingly better described by RL-Elo. In every case, RL-Elo did a better job of describing performance during the testing block, regardless of amount of training.

\section{Implications for Learning in General}

Subjects generally benefitted from training, a result that is not in itself surprising. However, several features of their performance are noteworthy. The extent of training corresponded to between four- and fivefold increases in the number of trials, but the gains observed in overall response accuracy (as captured by $\beta_{\emptyset}$ ) grew in an approximately linear fashion as a function of training. This pattern is consistent with an "accumulation of evidence" account of improving performance, as the rate at which new observations can contribute to the estimate of a mean is at most a factor of $\sqrt{n}$, given $\mathrm{n}$ observations. Since each subject displayed a different slope, we can infer that subjects differed in the information content they were able to extract from a single trial.

Dramatic individual differences were observed, and this has implications for experimental methodology. Most studies using animal models make use of extensive training, often consisting of thousands or tens of thousands of trials. Under an "evidence accumulation" framework, this is expected to drive subjects toward ceiling performance, which may serve to conceal individual differences that may have been more evident early in learning. This approach likely also suffers from diminishing returns. If subjects reliably show evidence of learning after mere dozens of trials, the time spent collecting thousands of trials of data might be better served by training subjects on multiple lists per day. When trying to understand the underlying learning process, having many examples of learning (e.g. multiple lists, each trained separately) generally yields more statistical power than having many trials in a single context (e.g. training a single list until asymptotic performance is reached).

Although the majority of studies of learning processes in non-human subjects focus on asymptotic behavior after extensive training, an undercurrent of results continues to suggest that subjects display detectable evidence of learning even from the earliest trials of training, provided one has sufficient evidence to separate the signal from the noise. These results are in no way limited to serial learning studies. Even at the height of associative learning theory's popularity, Estes (1960) argued persuasively that there was unambiguous evidence for single-trial learning. Pigeons foraging for rewards under concurrent operant schedules are sensitive to differential rewards after only one or two reward deliveries (Davison and Baum, 2003). Even goldfish learning to anticipate the timing of an uncomfortable shock display a peak in conditioned responding during the very earliest trials of training, long before "conditioning" is commonly thought to have had a chance to materialize (Drew et al., 2005). Single-trial learning effects have also been detected in the learning supported by the limbic system (Rutishauser et al., 2006), and cerebellum (Khilkevich et al., 2016). The possibility that subjects can measurably extract information from single experiences places important constraints on the learning models we should consider plausible, as well as 
on the varieties of neural circuitry that could implement such learning in the brain (Gallistel and Balsam, 2014).

Often, the existence of single-trial learning is used to make arguments about the optimality of learning. It is essential to distinguish, however, between error-free inference given a body of evidence and an effective heuristic whose performance has been optimized. Given the structure of the TI task, each subject is provided with sufficient information to make perfect responses on all subsequent trials after the first block of 6 training trials provided they fully understand the task structure. This type of "perfect serial inference" is essentially never seen, whether in humans or in non-human subjects, so the present evidence of serial learning during the first 6 trials is not evidence of a capacity for propositional logic. Instead, it represents the very earliest stages of a process by which organisms accumulate evidence. In addition to studying behavior that has reached a performance ceiling, there is much to be discovered by studying learning's earliest stages.

\section{Acknowledgments}

We would like to thank Yelda Alkan, Grant Spencer, Saani Borge, Aliza Gross, Katherine Liu, Krystian Loescher for their assistance in stimulus preparation and data collection.

\section{Funding}

This study was supported by NIH-MH081153 and NIH-MH111703 (VPF and HST).

\section{Author Contributions}

The study was designed by GJ, FM, HST, and VPF. Data were collected by FM, AM, and VPF. Data were analyzed by GJ. The paper was written by GJ, FM, AM, HST, and VPF.

\section{REFERENCES}

Carpenter, B., Gelman, A., Hoffman, M., Lee, D., Goodrich, B., Betancourt, M., Brubaker, M. A., Guo, J., Li, P., and Riddell, A. (2017). Stan: A probabilistic programming language. Journal of Statistical Software, 76:1-32.

Davison, M. and Baum, W. M. (2003). Every reinforcer counts: Reinforcer magnitude and local preference. Journal of the Experimental Analysis of Behavior, 8:95-129.

Drew, M. R., Zupan, B., Cooke, A., Couvillon, P. A., and Balsam, P. D. (2005). Temporal control of conditioned responding in goldfish. Journal of Experimental Psychology: Animal Behavior Processes, 31:31-39.

Estes, W. K. (1960). Learning theory and the new "mental chemistry.". Psychological Review, 67:207-223.

Gallistel, C. R. and Balsam, P. D. (2014). Time to rethink the neural mechanisms of learning and memory. Neurobiology of Learning and Memory, 108:136-144.

Jensen, G. (2017). Serial learning. In Call, J., Burghardt, G. M., Pepperberg, I. M., Snowdon, C. T., and Zentall, T., editors, APA Handbook of Comparative Psychology, pages 385-409. American Psychological Association, Washington, DC.

Jensen, G., Altschul, D., Danly, E., and Terrace, H. S. (2013). Transfer of a serial representation between two distinct tasks by rhesus macaques. PLOS ONE, 8:e70285.

Jensen, G., Munoz, F., Alkan, Y., Ferrera, V. P., and Terrace, H. S. (2015). Implicit value updating explains transitive inference performance: The betasort model. PLOS Computational Biology, 11:e1004523.

Jensen, G., Terrace, H. S., and Ferrera, V. P. (2019). Discovering implied serial order through model-free and model-based learning. Frontiers In Neuroscience, 13:Article 878. 
Khilkevich, A., Halverson, H. E., Caton-Josh, J. E., and Mauk, M. D. (2016). Links between single-trial changes and learning rate in eyelid conditioning. Cerebellum, 15:112-121.

Kumaran, D., Banino, A., Blundell, C., Hassabis, D., and Dayan, P. (2016). Computations underlying social hierarchy learning: Distinct neural mechanisms for updating and representing self-relevant information. Neuron, 92:1135-1147.

McElreath, R. (2020). Statistical Rethinking, Second Edition. CRC Press, Boca Raton, FL, USA.

Rutishauser, U., Mamelak, A. N., and Schuman, E. M. (2006). Single-trial learning of novel stimuli by individual neurons of the human hippocampus-amygdala complex. Neuron, 49:805-813.

Stoet, G. and Snyder, L. H. (2007). Extensive practice does not eliminate human switch costs. Cognitive, Affective, \& Behavioral Neuroscience, 7:192-197.

Tanner, N., Jensen, G., Ferrera, V. P., and Terrace, H. S. (2015). Inferential learning of serial order of perceptual categories by rhesus monkeys (Macaca mulatta). Journal of Neuroscience, 37:6268-6276.

Tromp, D., Meunier, H., and Roeder, J. J. (2015). Transitive inference in two lemur species (Eulemur macaco and Eulemur fulvus). American Journal of Primatology, 77:338-345.

Watkins, C. J. C. H. and Dayan, P. (1989). Q-learning. Machine Learning, 8:279-292.

Wynne, C. D. L. (1997). Pigeon transitive inference: Tests of simple accounts of a complex performance. Behavioural Processes, 39:95-112. 